Check for updates

Cite this: Mater. Adv., 2021, 2, 4694

Received 10th April 2021 Accepted 28th May 2021

DOI: $10.1039 / \mathrm{d} 1 \mathrm{ma} 00328 \mathrm{c}$

rsc.li/materials-advances

\title{
Can T-carbon serve as a Li storage material and a Li battery anode?†t
}

\author{
Li-Rong Cheng, Zheng-Zhe Lin, (D) * Xi-Mei Li and Xi Chen (D)*
}

\begin{abstract}
Carbon can form a wide variety of bulk structures. The possibility of carbon-based materials to be utilized in future energy applications has attracted extensive attention. T-carbon is a newly discovered diamond-like phase with an acetylene-bond supported hollow structure. This feature inspires us to consider its possibility of serving as a high-capacity Li storage material and a Li battery anode. Density functional theory calculations are performed to investigate the structure evolution, energy spectrum, stability, and electrode potential of Li-loaded T-carbon. The adsorption of T-carbon to Li atoms originates from the valence electrons of $\mathrm{Li}$ filling into the antibonding $\pi^{*}$ orbitals of acetylene bonds. The maximum theoretical Li capacity is three times that of graphite $\left(1116 \mathrm{~mA} \mathrm{~h} \mathrm{~g}^{-1}\right)$. At low Li density, too strong adsorption is disadvantageous to the release of $\mathrm{Li}$ and the discharge of the anode. At high $\mathrm{Li}$ density, Li atoms push against each other and fight against the adsorption of T-carbon. Such an advantageous issue leads to a decrease in anode potential. The significant difference of $\mathrm{Li}$ adsorption between low and high density makes a "dead zone" in which only half of the Li capacity could be useful for the discharge of the anode.
\end{abstract}

\section{Introduction}

As a fundamental element on the earth, carbon possesses the unique ability to form a variety of complex structures. With $\mathrm{sp}^{3}$, $\mathrm{sp}^{2}$, and $\mathrm{sp}$ hybridization, carbon-based structures exhibit chemical and biological diversity. Besides graphite and diamond in nature, people have synthesized many new carbon allotropes including fullerenes, ${ }^{1}$ carbon nanotubes, ${ }^{2}$ and graphene. ${ }^{3}$ With the development of synthesis technology, a large number of carbon allotropes with sp bonds or sp- $\mathrm{sp}^{2}$ and $\mathrm{sp}-\mathrm{sp}^{3}$ combinations have been obtained. ${ }^{4-8}$ Beyond that, theoretical predictions on new carbon crystalline phases, e.g. M-carbon, ${ }^{9}$ bct $\mathrm{C}_{4}$ carbon, ${ }^{10}$ penta-graphene, ${ }^{11}$ BC14 pentadiamond, ${ }^{12}$ BCO- $\mathrm{C}_{16},{ }^{13}$ and the graphyne family ${ }^{14}$ have been proposed. For a long time, carbon materials have been widely utilized in energy technology. Carbon matrices (amorphous carbon and many other forms) and carbides ${ }^{15,16}$ are always used as catalyst supports. The utilization of newly discovered carbon allotropes (e.g. graphdiyne) and carbides ${ }^{15,17}$ in emerging energy applications such as catalysis, Li-ion batteries, solar cells and hydrogen storage has been paid much attention. ${ }^{18-24}$

In 2011, T-carbon, a new diamond-like carbon allotrope (space group $F d \overline{3} m$ ), was theoretically proposed..$^{25}$ The structure

School of Physics and Optoelectronic Engineering, Xidian University, Xi'an 710071, China.E-mail:zzlin@xidian.edu.cn, xichen_phys@xidian.edu.cn

$\dagger$ PACS: 61.50.-f; 65.40.gk.

\# Electronic supplementary information (ESI) available. See DOI: 10.1039/d1ma00328c of T-carbon is constituted by substituting each atom in diamond by a carbon tetrahedron which connects with another tetrahedron by an acetylene bond. The calculated results show that T-carbon possesses a much lower density of $1.50 \mathrm{~g} \mathrm{~cm}^{-3}$ and a Vickers hardness of $61.1 \mathrm{GPa}$ (smaller than that of diamond (93.7 GPa) but comparable with that of cubic boron nitride). Recently, T-carbon has been successfully prepared by picosecond laser irradiation on carbon nanotubes ${ }^{26}$ and plasma-enhanced chemical vapor deposition. ${ }^{27}$ The potential applications of T-carbon in photocatalysis, solar cells, adsorption, energy storage, supercapacitors, aerospace materials, electronic devices are widely concerned. ${ }^{28,29}$ With a hollow structure, T-carbon is expected to be a promising material for Li storage. Its excellent carrier mobility ${ }^{29}$ and mechanical ductility $^{30}$ ensure favorable properties for use as the electrode in Li-ion batteries.

In this paper, density functional theory (DFT) calculations are employed to investigate the possibility of T-carbon serving as the material for Li storage and a Li battery anode. We carry out DFT-based Monte Carlo simulations to build the structure and energy spectrum of Li-loaded T-carbon. Molecular dynamics (MD) simulations are performed to confirm the structural stability. The convex hull of the energy spectrum is built to predict the structure evolution in the Li loading process. C-Li bonding and the mechanism of the change of $\mathrm{Li}$ adsorption strength in the loading process are analyzed. The anode potential exhibits a significant difference between the case of low and high Li density, which makes a "dead zone" in 
which only half of the Li capacity could be useful for the discharge of the anode. This work provides basic guidance for future studies on the possibility of utilizing other carbon allotropes in emerging energy applications.

\section{Computational methods}

DFT calculations are performed with the projector augmented wave (PAW) method ${ }^{31,32}$ as implemented in the Vienna ab initio simulation package (VASP). ${ }^{33-36}$ The electron exchange and correlation are described at the level of Perdew-Burke-Ernzerhof (PBE) functional. ${ }^{37}$ The correction of van der Waals interactions is treated by the DFT-D3 method with Becke-Jonson damping. ${ }^{38,39}$ A plane-wave basis set is used with a kinetic energy cutoff of $500 \mathrm{eV}$. The Brillouin-zone integration for the $1 \times 1 \times 1(2 \times 2 \times 2)$ T-carbon supercell is performed with an $8 \times 8 \times 8(4 \times 4 \times 4) \Gamma$-centered Monkhorst-Pack grid. For electronic structure calculations, a $25 \times 25 \times 25 \Gamma$-centered Monkhorst-Pack grid is used. The convergence of total energy is considered to be achieved until the total energy difference of two iterated steps is less than $10^{-5} \mathrm{eV}$. Geometry relaxations are performed until all the atomic forces are below $0.001 \mathrm{eV} \AA^{-1}$. The climbing image nudged elastic band method ${ }^{40-42}$ was used to find the minimum energy paths and barriers of Li migrations in T-carbon.

To verify the DFT parameters, we perform computations on graphite as the anode of $\mathrm{Li}$ battery. The main phases of Li-loaded graphite are $\mathrm{C}_{12} \mathrm{Li}_{1}$ (ESI, $\neq$ Fig. $\mathrm{S} 1$ (a), called as stage II in ref. 43) and $\mathrm{C}_{6} \mathrm{Li}_{1}$ (Fig. S1(b), ESI, $\ddagger$ called stage $\mathrm{I}$ in ref. 43). For the two phases, the calculated Li adsorption energy (eqn (1) and Fig. S1(c), ESI $\$)$ and the anode potential of graphite (see Section III.3. and Fig. S1(d), ESI $\ddagger$ ) are close to the results in ref. 43 .

For a certain number of $\mathrm{Li}$ atoms loading on the T-carbon supercell, the structure evolution is carried out using the Metropolis Monte Carlo method for the sampling of the energy spectrum. In every random move, each $\mathrm{Li}$ atom is moved with a displacement less than $1.5 \AA$, and then the structure is fully relaxed. Every relaxed configuration is accepted according to a Gibbs distribution on the DFT total energy within an effective temperature of $4000 \mathrm{~K}$. The structural stability is then verified by molecular dynamics (MD) simulations using the NoseHoover thermostat.

\section{Results and discussion}

\section{III.1. Preliminary understanding}

The structure of T-carbon is shown in the ESI, $\ddagger$ Fig. S2(a) (8 C atoms in one unit cell). T-carbon possesses a diamond-like architecture with acetylene bonds connecting neighboring $\mathrm{C}$ tetrahedrons. The energy bands and density of state (DOS) are shown in the ESI, F Fig. S2(b) and (c). It is known that the PBE functional always underestimates the bandgap. Thus, we further calculate electronic structures using the hybrid HeydScuseria-Ernzerhof (HSE06) functional. ${ }^{44,45}$ T-carbon presents a semiconducting feature with the calculated band gap of T-carbon being 2.23 (3.18) eV at the level of PBE (HSE06). Its hollow cage structure allows Li atoms to be stored in it.

To obtain a simple view of basic information, we first investigate $\mathrm{Li}$ adsorption in a $1 \times 1 \times 1 \mathrm{~T}$-carbon cell. To measure the strength of Li adsorption in T-carbon, we employ the Li adsorption energy

$$
E_{\text {ad }}=\left[E\left(\mathrm{~T}-\text { carbon- }-\mathrm{Li}_{n}\right)-E(\mathrm{~T} \text {-carbon })-n E(\mathrm{Li})\right] / n
$$

relative to the Li bulk. Here, $E$ (T-carbon) is the total energy of the T-carbon cell, $E(\mathrm{Li})$ is the energy per Li atom in the bulk bcc phase, and $E$ (T-carbon- $\left.\mathrm{Li}_{n}\right)$ is the total energy of the T-carbon cell with $n \mathrm{Li}$ atoms. For a single Li atom, the preferred position of $\mathrm{Li}$ is located in the C-cage (Fig. 1(a)). The structure of T-carbon- $\mathrm{Li}_{4}(\mathrm{C}: \mathrm{Li}=2: 1)$ is shown in Fig. 1(b), in which the Li-Li distance (2.4-3.3 $\AA$ ) is shorter or closer than that of the $\mathrm{Li}$ bulk (3.0 $\AA$ ). More Li atoms inserted into T-carbon may enlarge and destroy the $\mathrm{C}$ skeleton. So, this is considered as the state of maximum Li density. If T-carbon is used as the Li-ion battery anode (with the discharge process $\mathrm{Li} \rightarrow \mathrm{Li}^{+}+\mathrm{e}$ ), the maximum $\mathrm{Li}$ capacity is three times that of graphite $\left(1116 \mathrm{~mA} \mathrm{~h} \mathrm{~g}^{-1}\right)$. The structural stability of $1 \times 1 \times 1$-T-carbon- $\mathrm{Li}_{4}$ is confirmed by MD simulations at $300 \mathrm{~K}$ (ESI, $\$$ Movie S1). The evolution of temperature and total energy (Fig. 1(d)) fluctuating near the average values exhibits the stability of the system. We utilize Monte Carlo simulations to find the structure and energy spectrum of $\mathrm{Li}_{n}$ with $n=1-4$ (Fig. 1(c), where each line denotes the $\mathrm{Li}$ adsorption energy $E_{\text {ad }}$ of an individual structure). It can be seen that the $E_{\text {ad }}$ of the most stable structure gradually increases with the Li number $n$, showing a gradually saturated carrying of T-carbon to $\mathrm{Li}$ atoms. In $1 \times 1 \times 1$-T-carbon- $\mathrm{Li}_{4}$, $\mathrm{C}-\mathrm{Li}$ binding causes structure distortion making the lattice become a low-symmetry system and leading to a relaxation of the structure with extra energy decrease. Therefore, the most stable structure of $\mathrm{Li}_{4}$ possesses a lower energy than the most stable structure of $\mathrm{Li}_{3}$. The Li-rich T-carbon is then stabilized by the distortion effect.

\section{III.2. Li distribution and the convex hull of the energy spectrum}

To realize a more realistic spectrum of $\mathrm{Li}$ distribution in T-carbon, we turn to the structure search in a larger $2 \times 2 \times$ 2 T-carbon cell. The most stable structure of $\mathrm{Li}_{4}$ in $2 \times 2 \times 2$ T-carbon (Fig. 2(a)) exhibits partial segregation with more Li atoms aggregating together. The aggregated $\mathrm{Li}$ atoms may obtain lower energy. But for more Li atoms (Fig. 2(b) for $\mathrm{Li}_{8}$ and Fig. 2(c) for $\mathrm{Li}_{16}$ ), it is closer to a good distribution because the energy would be high if too many $\mathrm{Li}$ atoms aggregate in a small corner. The lattice of $2 \times 2 \times 2$-T-carbon- $\mathrm{Li}_{16}$ shows structure distortion with a tilted unit cell. The extra structure relaxation leads to the lowest energy and the T-carbon-Li system is then stabilized. This distortion can be known as the structural phase transition. For $2 \times 2 \times 2$-T-carbon-Li ${ }_{n}$ with $n \geq 16$, Li atoms are filled into this new phase. But the repulsion of denser $\mathrm{Li}$ causes an increase in the $\mathrm{Li}$ adsorption energy. As we inferred before, $1 \times 1 \times 1-\mathrm{Li}_{4}$ is the state of 

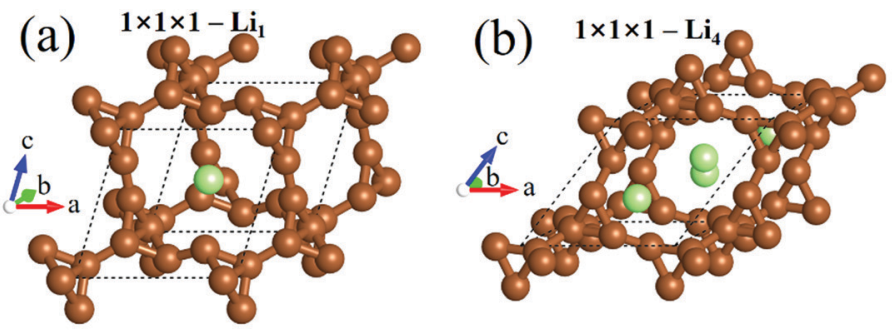

\section{(c)}

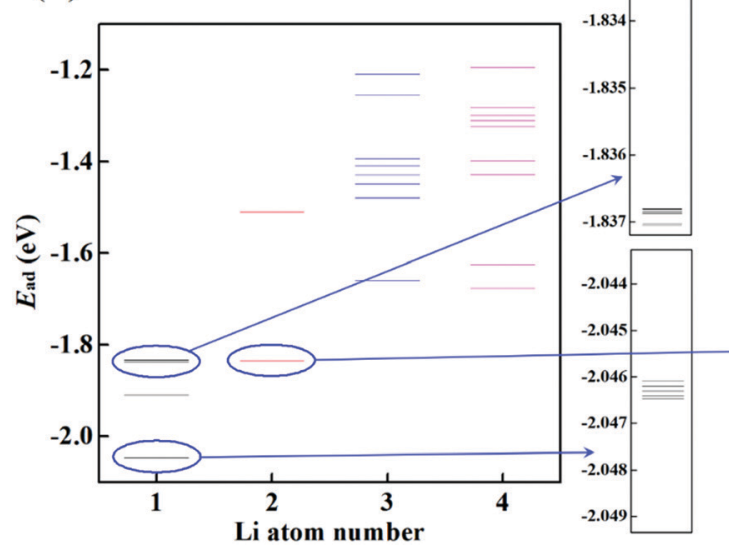

(d)
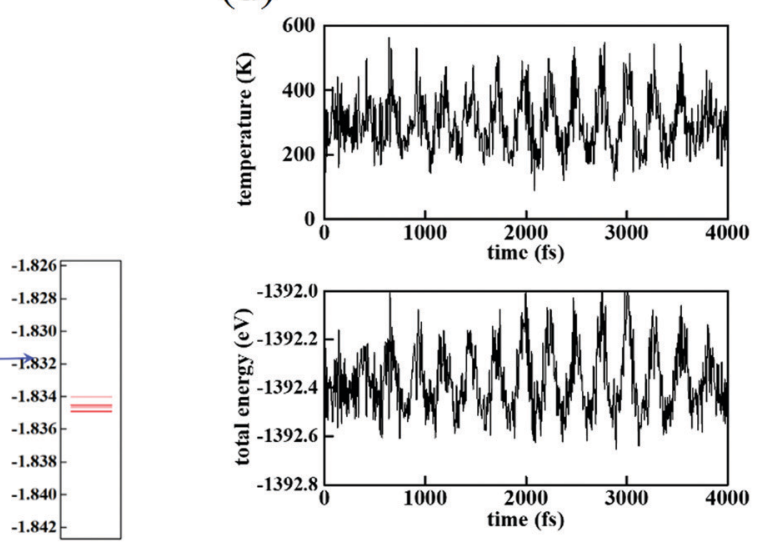

Fig. 1 (a) The most stable structure of $1 \times 1 \times 1 \mathrm{~T}$-carbon cell with $\mathrm{Li}_{1}$. (b) The most stable structure of $1 \times 1 \times 1 \mathrm{~T}$-carbon cell with $\mathrm{Li}_{4}$. (c) The energy spectrum of $1 \times 1 \times 1 \mathrm{~T}$-carbon cell with $\mathrm{Li}_{n}$. Some parts of $\mathrm{Li}_{1}$ and $\mathrm{Li}_{2}$ are enlarged. (d) The evolution of temperature and total energy in the MD simulation of $1 \times 1 \times 1 \mathrm{~T}$-carbon cell with $\mathrm{Li}_{4}$ at $300 \mathrm{~K}$.

maximum Li storage, and for a $2 \times 2 \times 2$ T-carbon cell the maximum is $\mathrm{Li}_{32}$ (Fig. 2(d)). MD simulations confirm the structural stability of $2 \times 2 \times 2-\mathrm{Li}_{32}$ (ESI, $\$$ Movie S2), with the evolution of temperature and total energy always fluctuating near the average values (Fig. 2(e)). A full energy spectrum for $\mathrm{Li}_{n}$ with $n=1-32$ is obtained by Monte Carlo simulations (Fig. 2(f)). It can be seen that the most stable structure of $\mathrm{Li}_{16}$ has the lowest energy, while the most stable energies of both sides $\mathrm{Li}_{1}$ and $\mathrm{Li}_{32}$ are high. In a complex energy spectrum, we are mainly concerned with the lowest energy structure of every $\mathrm{Li}_{n}$. The phases of $\mathrm{Li}_{n_{1}}$ (with an energy $E\left(n_{1}\right)$ ) and $\operatorname{Li}_{n_{2}}$ (with an energy $E\left(n_{2}\right)$ ) may mix with ratios $f$ and $1-f$, and the average energy per formula is

$$
\bar{E}=f E\left(n_{1}\right)+(1-f) E\left(n_{2}\right) .
$$

In comparison with the structure of the Li number $n=f n_{1}+$ $(1-f) n_{2}$, if $E(n)$ is lower than $\bar{E}$, the structure of $\operatorname{Li}_{n}$ would be the actually formed phase in the evolution of the T-carbon anode. However, in Fig. 2(f) the structures between $\mathrm{Li}_{1}$ and $\mathrm{Li}_{16}$ all have higher energy than $\bar{E}$ (see the red line). So, we infer that in the realistic charging process, the anode would be composed of a mixture of $\mathrm{Li}_{1}$ and $\mathrm{Li}_{16}$. Similarly, the structures between $\mathrm{Li}_{16}$ and $\mathrm{Li}_{32}$ all have higher energy than the average energy of $\mathrm{Li}_{16}$ and $\mathrm{Li}_{32}(f: 1-f)$. So in the subsequent charging process, the anode would be composed of a mixture of $\mathrm{Li}_{16}$ and $\mathrm{Li}_{32}$.

\section{III.3. Anode potential of Li-loaded T-carbon}

In this section, we will estimate the electrode potential of T-carbon serving as the anode of a Li battery. Generally, the ideal maximum electrode potential can be estimated by the change of Gibbs free energy in the process. In the last section, we inferred that in the charge or discharge process, the phases $2 \times 2 \times 2-\mathrm{Li}_{1}$ and $2 \times 2 \times 2-\mathrm{Li}_{16}$ (or $2 \times 2 \times 2-\mathrm{Li}_{16}$ and $2 \times 2 \times 2-\mathrm{Li}_{32}$ ) could coexist. For a phase $2 \times 2 \times 2-\mathrm{Li}_{n_{2}}$ changing into $2 \times 2 \times 2$-Li n $_{n_{1}}$ during discharge, the reaction on the side of the T-carbon anode is

$$
2 \times 2 \times 2-\mathrm{Li}_{n_{2}} \rightarrow 2 \times 2 \times 2-\mathrm{Li}_{n_{1}}+\left(n_{2}-n_{1}\right) \mathrm{Li}^{+}+\left(n_{2}-n_{1}\right) \mathrm{e} .
$$

Generally, the electrode potential is measured relative to the $\mathrm{Li}$ bulk. If the Li bulk is taken as the cathode, the reaction is

$$
\mathrm{Li}^{+}+\mathrm{e} \rightarrow \mathrm{Li}(\text { bulk). }
$$

The change of Gibbs free energy in the whole process $2 \times 2 \times$ $2-\mathrm{Li}_{n_{2}} \rightarrow 2 \times 2 \times 2-\mathrm{Li}_{n_{1}}+\left(n_{2}-n_{1}\right) \mathrm{Li}($ bulk $)$ is then

$\Delta G=G\left(2 \times 2 \times 2-\mathrm{Li}_{n_{1}}\right)+\left(n_{2}-n_{1}\right) G(\mathrm{Li}$ bulk $)-G\left(2 \times 2 \times 2-\mathrm{Li}_{n_{2}}\right)$.

Here, we approximately take free energy $G$ as the DFT total energy, and the anode potential then reads $V=\Delta G / e(e$ is the elementary charge).

The discharge of the T-carbon anode can be generally divided into three stages,

$$
\begin{gathered}
2 \times 2 \times 2-\mathrm{Li}_{32} \rightarrow 2 \times 2 \times 2-\mathrm{Li}_{16}+16 \mathrm{Li}^{+}+16 \mathrm{e}, \\
2 \times 2 \times 2-\mathrm{Li}_{16} \rightarrow 2 \times 2 \times 2-\mathrm{Li}_{1}+15 \mathrm{Li}^{+}+15 \mathrm{e},
\end{gathered}
$$




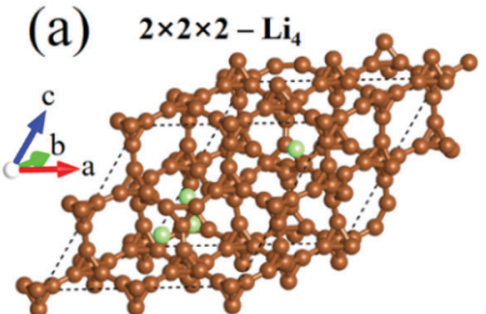

(b) $2 \times 2 \times 2-\mathbf{L i}_{8}$

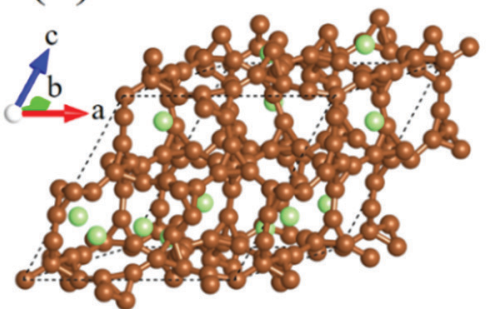

(e)
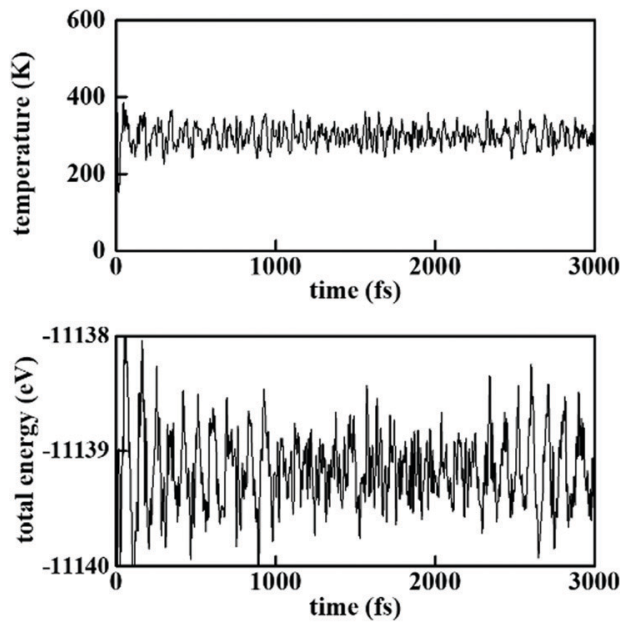

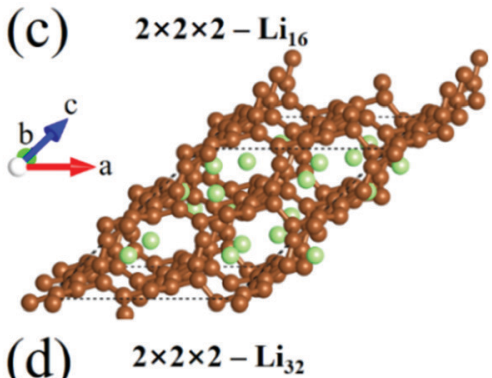

(d)

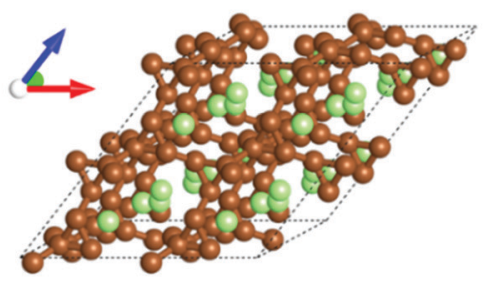

(f)

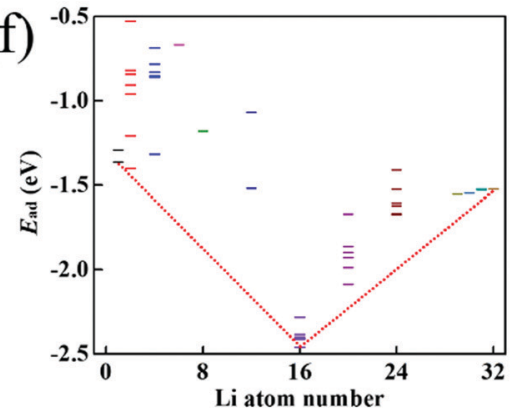

(g)

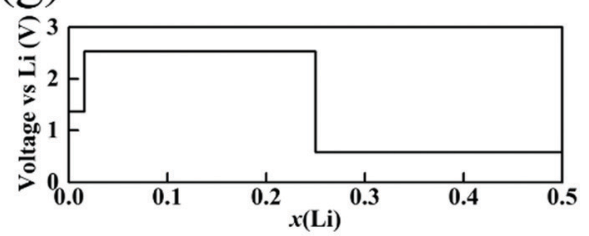

Fig. 2 The most stable structure of a $2 \times 2 \times 2 \mathrm{~T}$-carbon cell with (a) $\mathrm{Li}_{1}$, (b) $\mathrm{Li}_{8}$, (c) $\mathrm{Li}_{16}$, and (d) $\mathrm{Li} \mathrm{i}_{32}$. (e) The evolution of temperature and total energy in the MD simulations of a $2 \times 2 \times 2 \mathrm{~T}$-carbon cell with $\mathrm{Li}_{32}$ at $300 \mathrm{~K}$. (f) The energy spectrum and convex hull of a $2 \times 2 \times 2 \mathrm{~T}$-carbon cell with Li ${ }_{n}$. (g) The anode voltage of $\mathrm{T}$-carbon as the anode of Li battery.

and

$$
2 \times 2 \times 2-\mathrm{Li}_{1} \rightarrow \text { T-carbon }+\mathrm{Li}^{+}+\mathrm{e} .
$$

In the calculation results (Fig. $2(\mathrm{~g}), x(\mathrm{Li})=0-0.5$ is the ratio of $\mathrm{Li}: \mathrm{C}$ ), we can see that the anode voltages of these three stages are very different. Since the Li adsorption energy $E_{\text {ad }}$ in $2 \times 2 \times 2-\mathrm{Li}_{16}$ is much lower than the $E_{\text {ad }}$ in $2 \times 2 \times 2-\mathrm{Li}_{32}$, the process (6) is easy to proceed with because the energy goes down. This leads to a lower anode voltage. When the discharge reaches a certain degree, process (7) occurs thereupon. Since the $E_{\mathrm{ad}}$ in $2 \times 2 \times 2-\mathrm{Li}_{1}$ is much higher than the $E_{\text {ad }}$ in $2 \times 2 \times 2-\mathrm{Li}_{16}$, the process (7) proceeds with difficulty and then the anode voltage increases. The anode voltage of the first stage (6) is close to the anode voltage of graphite anode (about $0 \mathrm{~V}$ ). But the voltage of the second stage (7) is too high. Therefore, we infer that (7) is disadvantageous to the $\mathrm{Li}$ battery. The exploitable part is (6), in which the $\mathrm{Li}$ load is $x(\mathrm{Li})=$ $0.25-0.50$. Only a half of Li storage in T-carbon could be usable for battery discharge. Regarding $2 \times 2 \times 2-\mathrm{Li}_{16}$ as the substrate, the maximum load (16 more $\mathrm{Li}$ within) corresponds to a $\mathrm{Li}$ capacity of $487 \mathrm{~mA} \mathrm{~h} \mathrm{~g}^{-1}$, which is about 1.3 times the capacity of graphite.

\section{III.4. Electronic structure and the conductivity}

To use T-carbon as the anode of a Li battery, the electronic conductivity should be paid attention to. The widely used graphite is conductive, but pure T-carbon is a semiconductor with weaker conductivity. Li loading may enhance the conductivity of T-carbon. To investigate the effect of $\mathrm{Li}$, we calculate the projected density of states (PDOS). In $2 \times 2 \times$ $2-\mathrm{Li}_{1}, \mathrm{Li}$ bonds with $\mathrm{C}$ atoms and a part of $\mathrm{Li}$ electrons fill into C 2p orbitals (Fig. 3(a)). This leads to an energy level broadening near the Fermi level, and make some contribution to conductivity. A doping level locates at about $1 \mathrm{eV}$ above the Fermi level. With more Li atoms filling into T-carbon, 
(a)

$2 \times 2 \times 2-\mathbf{L i}_{1}$
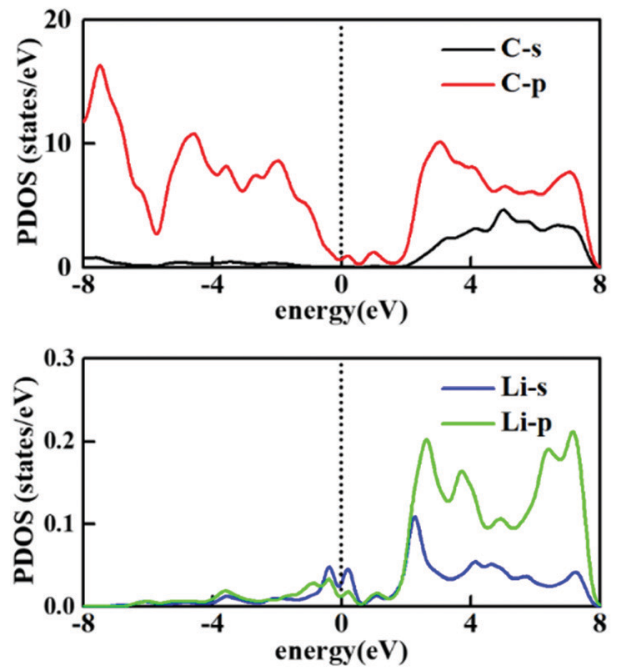

(b) $2 \times 2 \times 2-\mathbf{L i}_{32}$
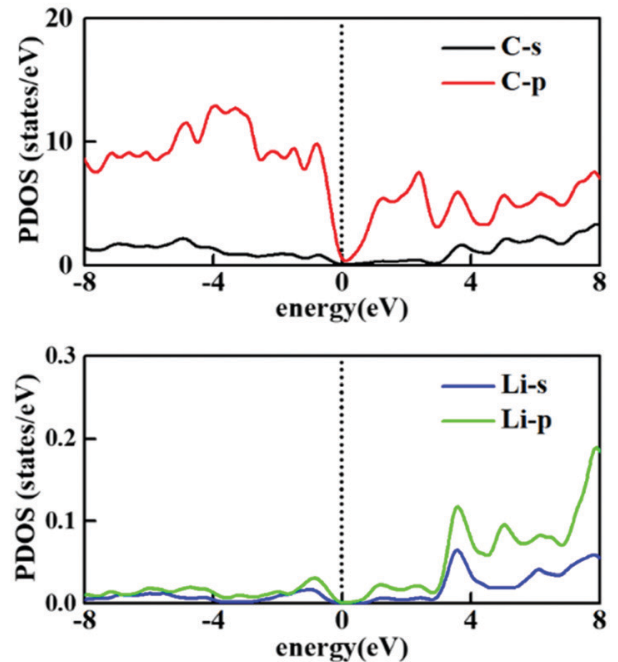

Fig. 3 PDOS of $2 \times 2 \times 2 \mathrm{~T}$-carbon cell with (a) $\mathrm{Li}_{1}$ and (b) $\mathrm{Li}_{32}$.

more electrons of $\mathrm{Li}$ fill into $\mathrm{C} 2 \mathrm{p}$ orbitals. At the maximum Li loading, i.e. $2 \times 2 \times 2-\mathrm{Li}_{32}$, the extra electrons just fill a whole band, and a new small bandgap appears at the Fermi level (Fig. 3(b)). There should gradually exhibit a change between the case of $\mathrm{Li}_{1}$ and $\mathrm{Li}_{32}$, which is discussed in the following text.

The electronic conductivity of bulk materials depends on electron-phonon relaxation and the electronic distribution in the bands. The conductivity tensor reads

$$
\overrightarrow{\vec{\sigma}}=\frac{e^{2}}{4 \pi^{3}} \int \tau\left(-\frac{\partial f}{\partial E}\right) \vec{V} \vec{V} d^{3} \vec{k}
$$

where $E$ is the electron energy, $\tau$ is the electron-phonon relaxation time, $\vec{V}=\partial E / \hbar \partial \vec{k}$ is the electron velocity, and $f=1 /\left(1+\exp \left[\left(E-E_{\mathrm{F}}\right) / k_{\mathrm{B}} T\right]\right)$ is the Fermi-Dirac distribution. We know that $\partial f / \partial E$ is a delta-like function that is sharp near the Fermi energy $E_{\mathrm{F}}$. In Fig. 4, we plot the density of states (DOS) in $2 \times 2 \times 2-\mathrm{Li}_{n}$ along with the outline of function $\partial \mathrm{f} / \partial E$ at $T=300 \mathrm{~K}$. For $2 \times 2 \times 2-\mathrm{Li}_{1}$ and $2 \times 2 \times 2-\mathrm{Li}_{8}$ (Fig. 4(a) and (b)), the contribution of DOS in the peak of $\partial f / \partial E$ is small, and the conductivity would be low. For $2 \times 2 \times 2-\mathrm{Li}_{16}$ and $2 \times 2 \times 2-\mathrm{Li}_{24}$ (Fig. 4(c) and (d)), DOS in the peak of $\partial f / \partial E$ is much larger and the conductivity would be higher. For $2 \times 2 \times 2-\mathrm{Li}_{32}$, since the electrons of Li fill a whole hand, a gap forms near the Fermi (a)

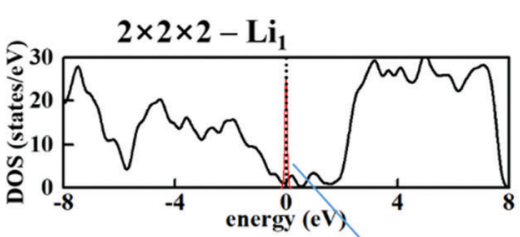

(b)

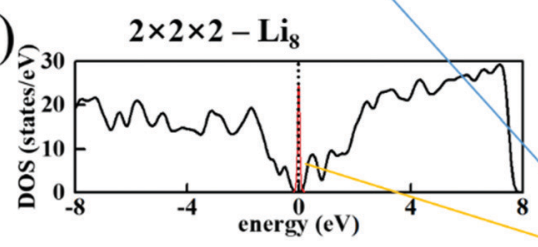

(c)

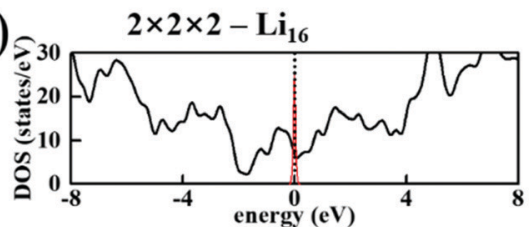

(d)

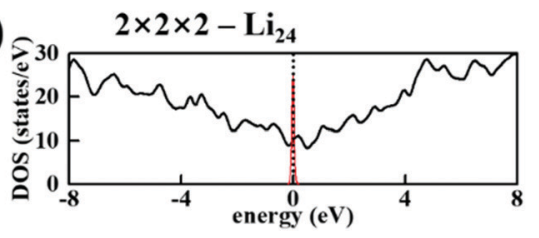

(e)

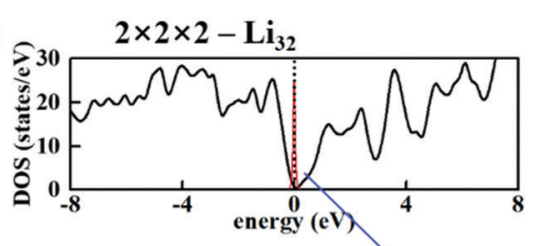

(f)
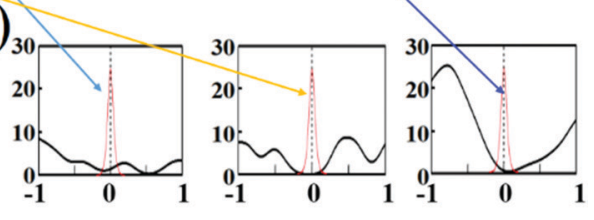

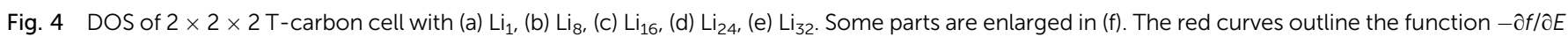
( $f$ is the Fermi-Dirac distribution function at $300 \mathrm{~K}$ ). 

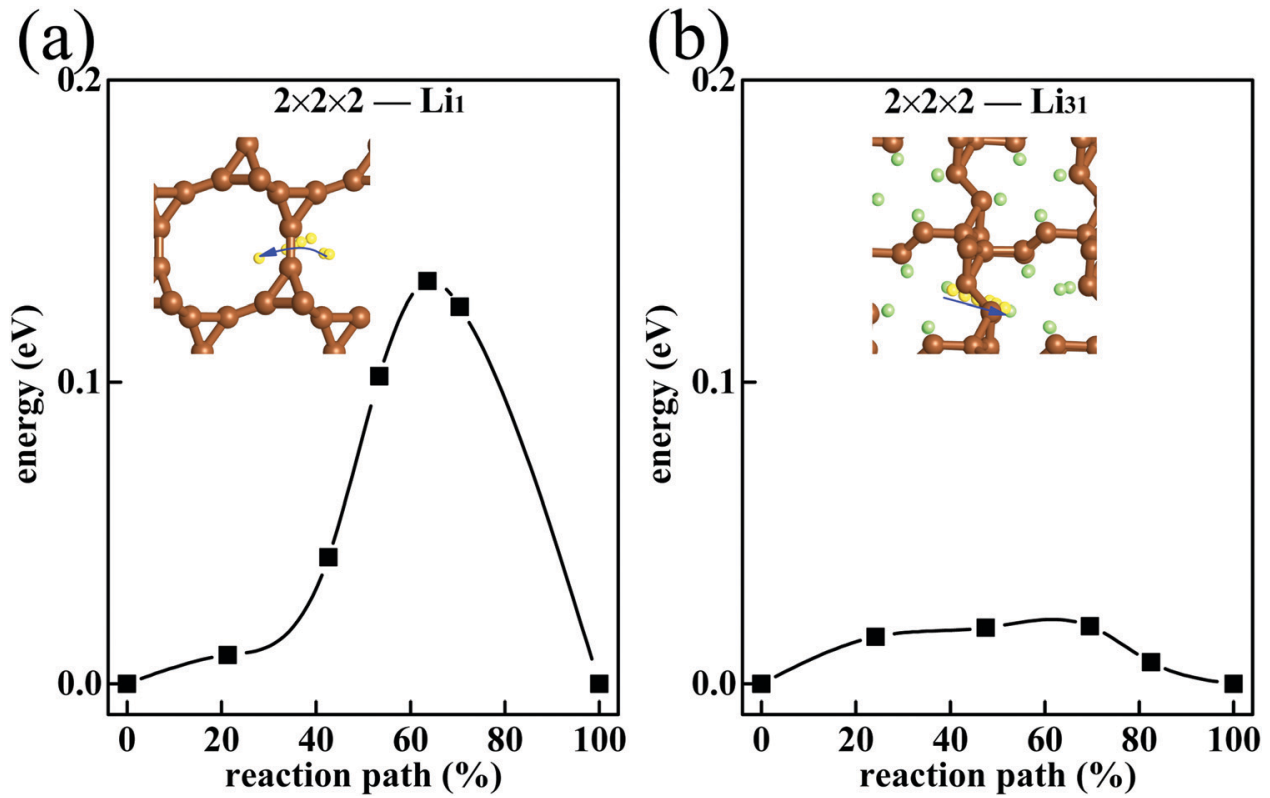

Fig. 5 The migration barrier of $\mathrm{Li}$ atom in $2 \times 2 \times 2 \mathrm{~T}$-carbon cell with (a) $\mathrm{Li}_{1}$ and (b) $\mathrm{Li}_{31}$. The migratory $\mathrm{Li}$ atom is shown in yellow.

energy and the electronic structure is semiconducting (a bandgap of $0.30 \mathrm{eV}$ ). The electron and hole density in the intrinsic semiconductor are

$$
n=\int_{\text {conduction band }} f \cdot \operatorname{DOS}(E) \mathrm{d} E
$$

and

$$
p=\int_{\text {valence band }}(1-f) \cdot \operatorname{DOS}(E) \mathrm{d} E
$$

respectively. The calculated full carrier density $n+p$ in $2 \times 2 \times$ 2- $\mathrm{Li}_{32}$ is $2.2 \times 10^{17} \mathrm{~cm}^{-3}$. Such a high carrier density ensures $2 \times 2 \times 2-\mathrm{Li}_{32}$ to be conductive. Overall, we infer that Li-rich T-carbon has enough carriers for the anode of the Li battery to be conductive.

To investigate the ionic conductivity, the migration barriers of $\mathrm{Li}$ in $\mathrm{T}$-carbon are investigated. In the initial charging process, low-concentration $\mathrm{Li}$ atoms form the $2 \times 2 \times 2-\mathrm{Li}_{1}$ phase, in which $\mathrm{Li}$ atoms can wander in the free space of T-carbon. Fig. 5(a) exhibits the migration process of the Li atom from one adsorption site to the neighboring site. The migration barrier $E_{\mathrm{b}}\left(\mathrm{Li}_{1}\right)=0.13 \mathrm{eV}$ is smaller than the Li diffusion barrier on pristine graphene (about $0.3 \mathrm{eV}$ ) ${ }^{46,47}$ graphene with a point defect (about $0.4-0.5 \mathrm{eV})^{46}$ and graphite (about $0.5 \mathrm{eV}$ ). ${ }^{48}$ This promises a faster Li diffusion in T-carbon.

In the final charging process, the system is saturated with $\mathrm{Li}$ (the $2 \times 2 \times 2-\mathrm{Li}_{32}$ phase). Thus, we consider the Li migration in the state of $2 \times 2 \times 2-\mathrm{Li}_{31}$ which is close to the saturation. Since $2 \times 2 \times 2-\mathrm{Li}_{31}$ is less filled than $2 \times 2 \times 2-\mathrm{Li}_{32}$, in $2 \times 2 \times 2-\mathrm{Li}_{31}$ a $\mathrm{Li}$ atom can migrate to a nearing vacancy. Such processes proceed successively, and then the $\mathrm{Li}$ atoms migrate. In $2 \times 2 \times$ $2-\mathrm{Li}_{31}$, a $\mathrm{Li}$ atom is affected by other $\mathrm{Li}$ in all directions, and the chemical environment is more isotropic. So, the $\mathrm{Li}$ migration barrier in $2 \times 2 \times 2-\mathrm{Li}_{31} E_{\mathrm{b}}\left(\mathrm{Li}_{31}\right)=0.02 \mathrm{eV}$ (Fig. 5(b)) is much lower than in T-carbon with low-concentration Li. In Li-filled T-carbon, the easier migration is beneficial to charging and discharging at high Li density. As the anode of the Li ion battery, $\mathrm{T}$-carbon is better than graphite with faster Li ion migration.

\section{Conclusions}

The combination of T-carbon and $\mathrm{Li}$ is systematically studied by DFT calculations and Monte Carlo simulations. T-carbon could serve as a $\mathrm{Li}$ storage and $\mathrm{Li}$ ion battery anode. Possible energy-favorable phases are $\mathrm{C}_{64} \mathrm{Li}, \mathrm{C}_{64} \mathrm{Li}_{16}$ and $\mathrm{C}_{64} \mathrm{Li}_{32}$. The maximum Li capacity is $1116 \mathrm{~mA} \mathrm{~h} \mathrm{~g}^{-1}$. The T-carbon-Li system prefers to form the mixture of these stable phases rather than other unstable phases. In the discharge process of the T-carbon anode, the transition $\mathrm{C}_{64} \mathrm{Li}_{32} \rightarrow \mathrm{C}_{64} \mathrm{Li}_{16} \rightarrow \mathrm{C}_{64} \mathrm{Li}$ causes a significant change in anode potential due to the difference of Li adsorption energy in these phases. The anode potential of the first stage $\mathrm{C}_{64} \mathrm{Li}_{32} \rightarrow \mathrm{C}_{64} \mathrm{Li}_{16}+16 \mathrm{Li}^{+}+16 \mathrm{e}$ is close to the anode potential of the graphite anode, while the anode potential of the second stage $\mathrm{C}_{64} \mathrm{Li}_{16} \rightarrow \mathrm{C}_{64} \mathrm{Li}+15 \mathrm{Li}^{+}+15 \mathrm{e}$ is high and disadvantageous to the Li battery. This leads to a "dead zone" in which only half of the Li capacity could be usable (with an effective capacity of $487 \mathrm{~mA} \mathrm{~h} \mathrm{~g}^{-1}$ ). Li storage in T-carbon contributes extra electrons into $\mathrm{C} \pi_{2 \mathrm{p}}$ orbitals and ensures its conductivity serving as an anode. Our work may provide guidance to the future study on the electrodes of $\mathrm{Li}, \mathrm{Na}$ or $\mathrm{K}$ ion batteries in emerging energy applications.

\section{Data availability}

The raw/processed data required to reproduce these findings cannot be shared at this time as the data also form part of an ongoing study. 


\section{Conflicts of interest}

The authors declare that they have no conflict of interest.

\section{Acknowledgements}

This work is supported by the Natural Science Basic Research Program of Shaanxi (No. 2021JM-117 \& 2021JQ-185), the Fundamental Research Funds for the Central Universities (No. XJS200503) and the Postdoctoral Research Project of Shaanxi Province (No. 2018BSHEDZZ68).

\section{References}

1 H. W. Kroto, J. R. Heath, S. C. O'Brien, R. F. Curl and R. E. Smalley, C60: buckminsterfullerene, Nature, 1985, 318, 162.

2 S. Iijima, Helical microtubules of graphitic carbon, Nature, 1991, 354, 56.

3 K. Novoselov, A. Geim, S. V. Morozov, D. Jiang, Y. Zhang and S. V. Dubonos, et al., Electric Field Effect in Atomically Thin Carbon Films, Science, 2004, 306, 666.

4 F. Diederich and M. Kivala, All-Carbon Scaffolds by Rational Design, Adv. Mater., 2010, 22, 803.

5 A. Hirsch, The era of carbon allotropes, Nat. Mater., 2010, 9, 868.

6 G. Li, Y. Li, H. Liu, Y. Guo, Y. Lia and D. Zhua, Architecture of graphdiyne nanoscale films, Chem. Commun., 2010, 46, 3256.

7 Y. Li, L. Xu, H. Liu and Y. Li, Graphdiyne and Graphyne: from Theoretical Predictions to Practical Construction, Chem. Soc. Rev., 2014, 43, 2572.

8 H. Yan, P. Yu, G. Han, Q. Zhang, L. Gu and Y. Yi, et al., HighYield and Damage-free Exfoliation of Layered Graphdiyne in Aqueous Phase, Angew. Chem., Int. Ed., 2019, 58, 746.

9 Q. Li, Y. Ma, A. R. Oganov, H. Wang, H. Wang and Y. Xu, et al., Superhard Monoclinic Polymorph of Carbon, Phys. Rev. Lett., 2009, 102, 175506.

10 K. Umemoto, R. M. Wentzcovitch, S. Saito and T. Miyake, Body-Centered Tetragonal $\mathrm{C}_{4}$ : a Viable $\mathrm{sp}^{3}$ Carbon Allotrope, Phys. Rev. Lett., 2010, 104, 125504.

11 S. Zhang, J. Zhou, Q. Wang, X. Chen, Y. Kawazoe and P. Jena, Penta-graphene: a new carbon allotrope, Proc. Natl. Acad. Sci. U. S. A., 2015, 112, 2372.

12 J.-T. Wang, C. Chen and H. Mizuseki, Body centered cubic carbon BC14: an all-sp ${ }^{3}$ bonded full-fledged pentadiamond, Phys. Rev. B, 2020, 102, 184106.

13 J.-T. Wang, H. Weng, S. Nie, Z. Fang, Y. Kawazoe and C. Chen, Body-Centered Orthorhombic $\mathrm{C}_{16}$ : a Novel Topological Node-Line Semimetal, Phys. Rev. Lett., 2016, 116, 195501.

14 D. Malko, C. Neiss, F. Viñes and A. Görling, Competition for Graphene: graphynes with Direction-Dependent Dirac Cones, Phys. Rev. Lett., 2012, 108, 086804.
15 H. Chen, Y. Hua, N. Luo, X. He, Y. Li and Y. Zhang, et al., Lithiation Abilities of SiC Bulks and Surfaces: a FirstPrinciples Study, J. Phys. Chem. C, 2020, 124, 7031.

16 Y. Shao, J. Liu, Y. Wang and Y. Lin, Novel catalyst support materials for PEM fuel cells: current status and future prospects, J. Mater. Chem., 2009, 19, 46.

$17 \mathrm{H}$. Vrubel and $\mathrm{X}$. $\mathrm{Hu}$, Molybdenum Boride and Carbide Catalyze Hydrogen Evolution in both Acidic and Basic Solutions, Angew. Chem., Int. Ed., 2012, 51, 12703.

18 Z. Zuo and Y. Li, Emerging Electrochemical Energy Applications of Graphdiyne, Joule, 2019, 3, 899.

19 Y. Fang, Y. Xue, L. Hui, H. Yu, Y. Liu and C. Xing, et al., In situ growth of graphdiyne based heterostructure: toward efficient overall water splitting, Nano Energy, 2019, 59, 591.

20 K. Srinivasu and S. K. Ghosh, Graphyne and Graphdiyne: promising Materials for Nanoelectronics and Energy Storage Applications, J. Phys. Chem. C, 2012, 116, 5951.

21 C. Li, J. Li, F. Wu, S.-S. Li, J.-B. Xia and L.-W. Wang, High Capacity Hydrogen Storage in Ca Decorated Graphyne: a First-Principles Study, J. Phys. Chem. C, 2011, 115, 23221.

22 J. He, N. Wang, Z. Cui, H. Du, L. Fu and C. Huang, et al., Hydrogen substituted graphdiyne as carbon-rich flexible electrode for lithium and sodium ion batteries, Nat. Commun., 2017, 8, 1172.

23 X. Ren, X. D. Li, Z. Yang, X. Wang, J. J. He and K. Wang, et al., Tailoring Acetylenic Bonds in Graphdiyne for Advanced Lithium Storage, ACS Sustainable Chem. Eng., 2020, 8, 2614.

24 X. D. Li, N. Wang, J. J. He, Z. Yang, Z. Y. Tu and F. H. Zhao, et al., Designing the efficient lithium diffusion and storage channels based on graphdiyne, Carbon, 2020, 162, 579.

25 X.-L. Sheng, Q.-B. Yan, F. Ye, Q.-R. Zheng and G. Su, T-Carbon: a Novel Carbon Allotrope, Phys. Rev. Lett., 2011, 106, 155703.

26 J. Zhang, R. Wang, X. Zhu, A. Pan, C. Han and X. Li, et al., Pseudo-topotactic conversion of carbon nanotubes to T-carbon nanowires under picosecond laser irradiation in methanol, Nat. Commun., 2017, 8, 683.

27 K. Xu, H. Liu, Y.-C. Shi, J.-Y. You, X.-Y. Ma and H.-J. Cui, et al., Preparation of T-carbon by plasma enhanced chemical vapor deposition, Carbon, 2020, 157, 270.

28 G. Qin, K.-R. Hao, Q.-B. Yan, M. Hu and G. Su, Exploring T-carbon for energy applications, Nanoscale, 2019, 11, 5978.

29 P.-P. Sun, L. Bai, D. R. Kripalani and K. Zhou, A new carbon phase with direct bandgap and high carrier mobility as electron transport material for perovskite solar cells, $n p j$ Comput. Mater., 2019, 5, 9.

30 L. Bai, P.-P. Sun, B. Liu, Z. Liu and K. Zhou, Mechanical behaviors of T-carbon: a molecular dynamics study, Carbon, 2018, 138, 357.

31 P. E. Blöchl, Projector augmented-wave method, Phys. Rev. B: Condens. Matter Mater. Phys., 1994, 50, 17953.

32 G. Kresse and D. Joubert, From ultrasoft pseudopotentials to the projector augmented-wave method, Phys. Rev. B: Condens. Matter Mater. Phys., 1999, 59, 1758.

33 G. Kresse and J. Furthmüller, Efficient iterative schemes for $a b$ initio total-energy calculations using a plane-wave basis 
set, Phys. Rev. B: Condens. Matter Mater. Phys., 1996, 54, 11169.

34 G. Kresse and J. Furthmüller, Efficiency of ab-initio total energy calculations for metals and semiconductors using a planewave basis set, Comput. Mater. Sci., 1996, 6, 15.

35 G. Kresse and J. Hafner, Ab initio molecular dynamics for liquid metals, Phys. Rev. B: Condens. Matter Mater. Phys., 1993, 47, 558.

36 G. Kresse and J. Hafner, $A b$ initio molecular-dynamics simulation of the liquid-metal-amorphous-semiconductor transition in germanium, Phys. Rev. B: Condens. Matter Mater. Phys., 1994, 49, 14251.

37 J. P. Perdew, K. Burke and M. Ernzerhof, Generalized gradient approximation made simple, Phys. Rev. Lett., 1996, 77, 3865.

38 S. Grimme, J. Antony, S. Ehrlich and H. Krieg, A consistent and accurate $\mathrm{ab}$ initio parametrization of density functional dispersion correction (DFT-D) for the 94 elements $\mathrm{H}-\mathrm{Pu}$, J. Chem. Phys., 2010, 132, 154104.

39 S. Grimme, S. Ehrlich and L. Goerigk, Effect of the damping function in dispersion corrected density functional theory, J. Comput. Chem., 2011, 32, 1456.

40 G. Mills and H. Jónsson, Quantum and thermal effects in $\mathrm{H} 2$ dissociative adsorption: evaluation of free energy barriers in multidimensional quantum systems, Phys. Rev. Lett., 1994, 72, 1124.
41 G. Mills, H. Jónsson and G. K. Schenter, Reversible work transition state theory: application to dissociative adsorption of hydrogen, Surf. Sci., 1995, 324, 305.

42 G. Henkelman, B. P. Uberuaga and H. Jónsson, A climbing image nudged elastic band method for finding saddle points and minimum energy paths, J. Chem. Phys., 2000, 113, 9901.

43 K. Persson, Y. Hinuma, Y. S. Meng, A. van der Ven and G. Ceder, Thermodynamic and kinetic properties of the Li-graphite system from first-principles calculations, Phys. Rev. B, 2010, 82, 125416.

44 J. Heyd, G. E. Scuseria and M. Ernzerhof, Hybrid functionals based on a screened Coulomb potential, J. Chem. Phys., 2003, 118, 8207-8215.

45 J. Heyd, G. E. Scuseria and M. Ernzerhof, Erratum: hybrid functionals based on a screened Coulomb potential, J. Chem. Phys., 2006, 124, 219906.

46 L.-J. Zhou, Z. F. Hou and L.-M. Wu, First-Principles Study of Lithium Adsorption and Diffusion on Graphene with Point Defects, J. Phys. Chem. C, 2012, 116, 21780.

47 X. F. Fan, W. T. Zheng and J. L. Kuo, Adsorption and diffusion of Li on pristine and defective graphene, ACS Appl. Mater. Interfaces, 2012, 4, 2432.

48 K. Toyoura, Y. Koyama, A. Kuwabara, F. Oba and I. Tanaka, First-principles approach to chemical diffusion of lithium atoms in a graphite intercalation compound, Phys. Rev. B: Condens. Matter Mater. Phys., 2008, 78, 214303. 